\title{
Prospective evaluation of circumferential and longitudinal strain in asymptomatic children with dual ventricles who underwent single ventricle repair: comparison to single LV, single RV and normal hearts
}

\author{
Ramkumar Krishnamurthy ${ }^{2}$, Cory V Noel ${ }^{1 *}$, Amol Pednekar ${ }^{3}$, Ricardo Pignatelli', Rajesh Krishnamurthy ${ }^{2}$ \\ From 18th Annual SCMR Scientific Sessions \\ Nice, France. 4-7 February 2015
}

\section{Background}

Rarely, patients with normally sized RV and LV will undergo total cavopulmonary connection (TCPC) due to the complexity of their intracardiac anatomy giving them a dual ventricle (DV) for a single cardiac output. The ventricular function in this unique physiology compared to SRV, SLV and normal hearts remains poorly understood, with few studies performed ${ }^{1-3}$. In this study, we perform a comprehensive comparison of global and regional strain in both the circumferential $\left(\varepsilon_{\mathrm{cc}}\right)$ and longitudinal $\left(\varepsilon_{\mathrm{L}}\right)$ dimensions to conventional SV hearts and normal hearts.

\section{Purpose}

In normal subjects and asymptomatic patients with DV (LV and RV calculated independently), SLV and SRV after TCPC, to compare:

1) Global $\varepsilon_{\mathrm{cc}}$ and $\varepsilon_{\mathrm{L}}$ strain,

2) Regional circumferential and longitudinal strains at free wall $\left(\varepsilon_{\mathrm{cc}-\text { free, }}, \varepsilon_{\mathrm{L}-\text { freee }}\right)$ and septum $\left(\varepsilon_{\mathrm{cc}-\text { sept}}, \varepsilon_{\mathrm{L}-\text { sept }}\right)$,

3) $\varepsilon_{\mathrm{cc}}$ and $\varepsilon_{\mathrm{L}}$ across the ventricle from apex to base.

\section{Methods}

We performed a prospective analysis of 23 subjects (7 normals age in years: $11.8+/-3.1,5$ DV age: $12.4+/-2.7,6$ SRV age: $11.4+/-2.3,5$ SLV age: $12.6+/-4.2$ ).

${ }^{1}$ Pediatric Cardiology, Baylor College of Medicine, Houston, TX, USA

Full list of author information is available at the end of the article

\section{Acquisition Protocol}

Strain information was acquired at three short axis slices at basal, mid-cavity, and apical locations in all 123 subjects in a 1.5T MRI scanner (Philips Acheiva) using: a) Complementary Spatial Modulation of Magnetization (CSPAMM) images: Used for generating $\varepsilon_{\mathrm{cc}}$; and b) Fast-Strain Encoded (fSENC) images: Used for generating $\varepsilon_{\mathrm{L}}$.

\section{Data Analysis}

$\varepsilon_{\mathrm{cc}}$ and $\varepsilon_{\mathrm{L}}$ were calculated from SAX slices using Diagnosoft ${ }^{\mathrm{TM}}$. The ventricular regions at each slice were assigned based upon the AHA 16 segment model (fig. 1). $\varepsilon_{\text {cc-sept, }} \varepsilon_{\mathrm{L}-\text { sept }}, \varepsilon_{\mathrm{cc}-\text { free, and }} \varepsilon_{\mathrm{L}-\text { free }}$ were also calculated for each slice and compared.

\section{Results}

1.) Compared to normals, there is a significant reduction in global $\varepsilon_{\mathrm{cc}}$ at all ventricular levels of DV patients (fig 2).

2.) Compared to normals, there is a significant reduction in global $\varepsilon_{\mathrm{L}}$ in mid-ventricular and apical locations of DV patients.

3.) The $\varepsilon_{\mathrm{cc}}$ of the LV of DV patients consistently lower than SLV for global and regional calculations.

4.) In the same DV patient at the basal location, the $\varepsilon_{\text {cc-free }}$ was higher in the RV $(75+/-42 \%)$, with the $\varepsilon_{\mathrm{L} \text {-free }}$ being higher in the LV $(25+/-10 \%)$.

5.) Global $\varepsilon_{\mathrm{L}}$ progressed from base to apex in all groups. 

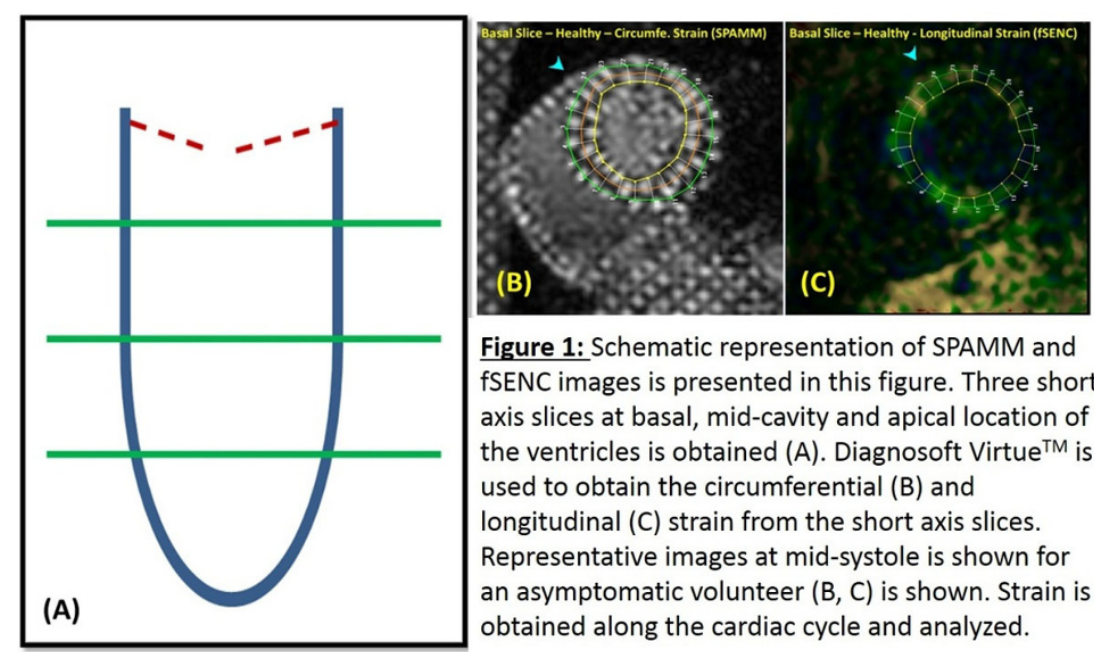

Figure 1: Schematic representation of SPAMM and fSENC images is presented in this figure. Three short axis slices at basal, mid-cavity and apical location of the ventricles is obtained (A). Diagnosoft Virtue ${ }^{T M}$ is used to obtain the circumferential (B) and longitudinal (C) strain from the short axis slices. Representative images at mid-systole is shown for an asymptomatic volunteer $(B, C)$ is shown. Strain is obtained along the cardiac cycle and analyzed.

Figure 1
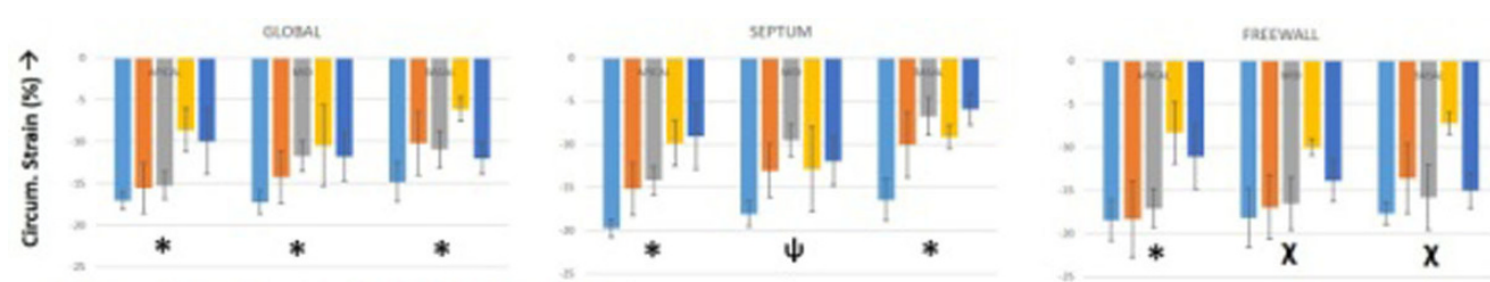

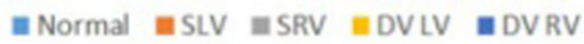
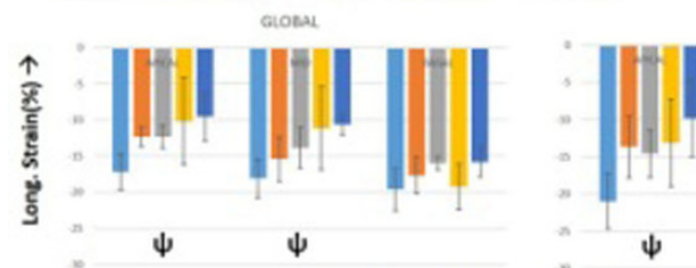

strmus
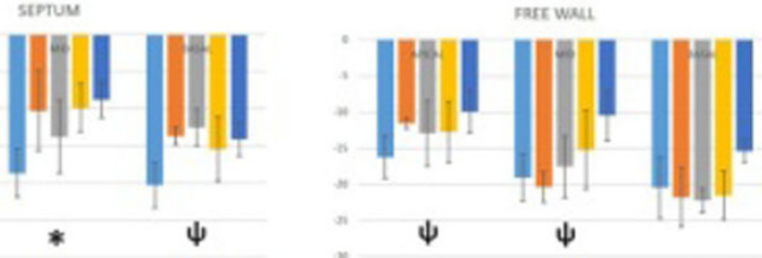

$\rightarrow p<0.05$ for Normal VS. DV IV, Normal vs. DV RV; $\psi \rightarrow p<0.05$ for Normal vs. DV RV; $x \rightarrow p<0.05$ for Normal vs. DV IV (paired Student's t-test)

Figure 2 Bar plots comparing the longitudinal $\left(\varepsilon_{\llcorner}\right)$and circumferential $\left(\varepsilon_{c c}\right)$ strain values in a pediatric population betweennormal subjects, patients with dual ventricles wih single ventricular repair and patients with systemic single ventricles. We demonstrate a significant reduction in both $\varepsilon_{\llcorner}$and $\varepsilon_{c c}$ compared to normal.

\section{Conclusions}

Strain values of the RV and LV in DV patients demonstrate significant differences compared to normal subjects. Additionally, the LV of DV patients had lower strain values than the SLV patients. The differences in the RV and LV within the same DV patient suggest inherent differences in ventricular biomechanics in this unique physiology. The shared workload of the LV and $\mathrm{RV}$ for a single cardiac output may contribute to their lower strain values.

\section{Authors' details}

'Pediatric Cardiology, Baylor College of Medicine, Houston, TX, USA.

${ }^{2}$ Radiology, Texas Children's Hospital, Houston, TX, USA. ${ }^{3}$ Clinical Science,

Philips Healthcare, Houton, TX, USA.

Published: 3 February 2015

\section{References}

1. Circ $98: 330$.

2. JASE 25:11, 1222.

3. Con.HD 7:16

doi:10.1186/1532-429X-17-S1-P212

Cite this article as: Krishnamurthy et al: Prospective evaluation of circumferential and longitudinal strain in asymptomatic children with dual ventricles who underwent single ventricle repair: comparison to single LV, single RV and normal hearts. Journal of Cardiovascular Magnetic Resonance 2015 17(Suppl 1):P212. 